\title{
The Study on the Translation of Metaphor in Cognitive Perspective
}

\author{
Fuyuan $\operatorname{Lei}^{1}$, PingYi ${ }^{1 *}$ \\ ${ }^{1}$ School of Foreign Language, Chengdu University of Traditional Chinese Medicine, Chengdu, Sichuan 610072, China \\ *Corresponding author.Email: 904026750@qq.com
}

\begin{abstract}
The study of metaphor has gone through more than two thousand years. The traditional method of metaphor study was confined to the field of rhetoric. In this method, metaphor was considered as a decorative part of the language. Modern metaphor studies have found that metaphor is not just a rhetoric device, but also a cognitive phenomenon. People use metaphor to cognize, to think and to describe experiences of ordinary life. During the communication of people in different cultural backgrounds, metaphor is often used, so the translation of metaphor is surely important. In this article, based on the cognitive perspective, there are three kinds of methods of metaphor translation, image-retaining translation, image-changing translation and image-omitting translation. Each of the translation method has its own situation for using. The translator should make a flexible method in the concrete context and never just stick to the rules.
\end{abstract}

Keywords: Metaphor, cognitive, metaphor translation

\section{INTRODUCTION}

Generally speaking, the most difficult part of translation is the difference between the two cultures. In culture A, one thing can be understood without any explanation, while in culture B, it took the translator great effort to make it clear. The communication in culture is the important part of translation, which cannot be focused only on the transfer between languages. Therefore, those culture-based words or phrases, such as metaphors, require to be thought twice in translation.

Our life is filled with metaphor. For instance, a piece of furniture has legs, the mountain has foot and the trees have crowns. What's more, the same image in different languages can have different connotations. For example, "dog" in Chinese always implies repulsion, but in English, "love me, love my dog", "top dog", "lucky dog", all of which show the image of dog in a positive way. How to translate metaphor appropriately is one of the important problems in translation research.

\section{METAPHOR}

The word "metaphor" comes from Greek metapherein, meaning "to transfer" or to "carry over". In Encyclopedia Britannia, metaphor is "a figure of speech that implies comparison between two unlike entities, as distinguished from simile, an explicit comparison signaled by the words 'like' or 'as"' [1]. Here it describes metaphor in rhetoric way, which is traditional. This point of view of Metaphor can be traced back to Aristotle, with the start of his two works the Poetics and Rhetoric, in which the formation and rhetoric function of metaphor have been much discussed. The Aristotelian definition of metaphor appears in the Poetics: "Metaphor consists in giving the thing a name that belongs to something else; the transference being either from genus to species, from species to genus, or from species to species, or on the ground of analogy" [2]. Aristotle believes metaphor is primarily ornamental, a decorative additive to language.

In 1930 s, the research of metaphor shift from the rhetoric tradition to semantic view. In I.A. Richards book The Philosophy of Rhetoric, he put forward the Interaction Theory of Metaphor. On the basis of that, Black developed the Interaction Theory, which made a breakthrough that metaphor is understood and interpreted from the semantic view rather than merely from the rhetoric tradition. The semantic approach pays much attention to the linguistic analysis from the perspective of semantic. "In semantic view, metaphor is researched as a semantic phenomenon in the level of sentence rather than a figure of speech in the level of word" [3].

With the development in various fields, the scholars wanted to understand metaphor further. Therefore, they adopted various views from many scientific areas, such as psychology, ethnography, anthropology or cultural communication, to re-analyze metaphor and hope to deepen the understanding of the nature of metaphor. Among these fields, cognitive perspective seems to be the current dominant way in the research of metaphor.

The book, Metaphors We Live by, written by George Lakoff and Mark Johnson, was published in 1980s, which was a representative work in cognitive study of metaphor. It is claimed that metaphor is conceptual and that many of our actions are based on metaphorical conception. Their argument is that human language is constructed in a metaphorical conceptual system. Metaphor is not to be 
seen as a purely linguistic phenomenon, but a cognitive phenomenon. Their argument is "that metaphor is pervasive in everyday life, not just in language but in thought and action," and "our ordinary conceptual system, in terms of which we think and act, is fundamentally metaphorical in nature" [4]. We do not just employ and construct metaphors but live through them. Our ordinary conceptual system by means of which we live, think and act is fundamentally metaphorical in nature. The essence of metaphor is understanding and experiencing one kind of thing in terms of another [5]. Take the following metaphors as example, "Time is money" and "love is journey". The basic matter in these metaphors are time and love, however, since these concepts are not so clearly explained in their own terms, in order to meet the requirement of our daily communication, they are experienced and defined as money and journey.

As metaphor considered as not only a rhetorical device but also a cultural phenomenon, Newmark classified different types of metaphors in relation to their contextual factors and translation procedures. He designed a"A Diagram of Metaphors and Their Translations" presented in Table 1 [6].

\begin{tabular}{ll} 
Table 1. 'A Diagram of Metaphors and Their Translations' by P.Newmark. \\
\hline Type of metaphor & Translation procedure \\
\hline Dead & Same image \\
Cliché & Different image \\
Standard & Reduce to sense \\
Original & Adapt images \\
Metonym & Sense plus image \\
Weakened & Simile \\
Redundant & Deletion
\end{tabular}

According to the table of translation procedure, Newmark pointed out that a translator can replace, reproduce, even delete the images of metaphors in translation.

Above all, metaphor is not merely a matter of words and not just a rhetorical device. Metaphor cannot be separated from thought. It is a cognitive device capable of communicating insight and truth. It is a tool for people to comprehend the world around. Since metaphor is shaped by culture, our translation of metaphor should be based on the cognitive perspective, where metaphors must be looked at as cognitive device representing how people conceptualize and record their experiences, attitudes and practices. However, there is one thing translators need to pay attention to. That is "translation of metaphor has been treated as part of the more general problem of 'untranslatability.' This trend builds on the fact that metaphors in general are associated with 'indirectness,' which in turn contributes to the difficulty of translation" [7]. Because no two languages in the world having the same history and same prosody, it is impossible to recreate the literary form and the prosody of a literary work composed in one culture in the language of another culture. And different cultures hold different knowledge, experience and beliefs. At the same time, metaphor bears high contents of culture. Therefore, metaphor translation becomes especially difficult and tough.

Though the exactly same translation between source language and target language is impossible, the effective metaphor translation still can be achieved.

\section{WAYS OF TRANSLATION}

Since metaphors are related to different cultural domains, this implies that the translator has to conceptualize things on behalf of the target language readers and has to look for a similar cognitive equivalence in target culture and in target language. The more the source language and target language cultures conceptualize experience in a similar way, the easier the task of translation will be. Although English and Chinese are two different languages, the metaphors within the two languages still have some same or similar conceptual parts.

In the cognitive perspective, metaphor is a conceptual system and through which we think and act. Applying the theory to the metaphor translation, we can categorize the approach of translation into the following three kinds.

\subsection{Image-retaining Translation}

First is retaining the image. The ideal way of translation is to retain the original image in target language without confusion and misunderstanding. It happens when metaphors expressing a small number of ideas shared by the two languages and expressed by similar expressions. Therefore, meaning of the translated version is basically equivalent to that of the source text. But the cognitive matter in both languages should be close enough.

This kind of translation keeps the style and cultural connotation in source language, and the original meaning surfer least of all loss during the process of translation, such as "castle in the air" (kong zhong lou ge), "to add fuel to the flames" (huo shang jiao you), "sunny smile" (yang guang ban de xiao rong), "give the green light" (kai lv deng) and "to trim the sail to the wind" (jian feng shi duo). These phrases are widely used, and we can find that how similar Chinese and English can be. In these phrases, the translator does not need to change another way to make them clear. There happens to be similar Chinese correspondences for them. And in the following sentence: "All the world is a stage, and all men and women merely players; they have their exits and their entrances." (quan shi jie shi yi ge da wu tai, suo you de nan nan nv nv bu guo shi xie yan yuan, ta men dou you xia chang de shi hou, ye dou you shang chang de shi hou). In this sentences, there is no need to change the original image "stage" in the translation. People both in Chinese culture and English culture share the same experience and the metaphor reveal the experience in words.

One of the advantages of image-retaining translation is the introduction of new concepts, new expressions and new sentence structures from the source language into the target 
language. For example, "arm to the teeth" (wu zhuang dao ya chi), "the Trojan Horse" (te luo yi mu ma), "Zhi lao hu" (paper tiger) and "Diu lian" (lose face). The translator has to pay attention to that, sometimes, the images in source language do have similar words in target language, but the two have totally different connotations. In this situation, sticking to image-retaining translation will lead to mistake. For example, "dead dog" can not be translated into "si gou", which actually means something without any value in English. And in some cases, just retaining the image is not enough, which need a little explanation. For instance, "Like a son of Bachus, he can drink up two bottle of Whiskey out of a breath" (ta jian zhi jiu xiang jiu shen ba ke si de er zi, neng yi kou he guang liang ping wei shi ji). The literal translation of Bachus cannot show the connotation, because Chinese people are not familiar with the name, so an additional explanation "jiu shen" is needed to make it easy for readers.

\subsection{Image-changing Translation}

The second method is changing the image. Different in history, customs and natural environment, people in different culture would conceptualize a same thing in different ways. That is the big gap between the source and target cognitive environment. In metaphor translation, it means that one image has different connotations in two languages. In the case, the translator should find another image in target language that can arouse same association to take place of the original one.

For example, "a black sheep" (hai qun zhi ma), "a cat on hot bricks" (re guo shang de ma yi) and "as cool as cucumber" (tai ran zi ruo). In these phrases, the original image is sheep, cat and cucumber. If the translator keeps the image and translates them literally, the Chinese readers would get confused. Usually, we do not associate these things to the meanings that the English sentences are showing, especially the last phrase "as cool as cucumber". If we translate it into "huang gua ban leng jing" is a totally ridiculous phrase.

And in a sentence, "It was diamond cut diamond when the two men met because they were both so sure their own ideas were right". (zhe liang ren dou jian xin zi ji de zhu zhang, yin ci ta men xiang yu ding shi yi chang qi feng dui shou de han zhan). We are used to compare lifelong marriage and love to diamond, but seldom apply diamond to the metaphor of competitor. Here in English, "diamond cut diamond" means both of the competitors are equal in willpower and fortitude. Simply translating into "zuan shi zhi zhan" can not make Chinese readers understand. In this situation, it is the best choice for translators to find another equivalent expression in target language. So "qi" is chosen to replace the original image "diamond".

In the above examples, the images in the source language are replaced with the target language images that contain the same connotation. As a result, the version can easily get across to the target language readers. However, we should be cautious that the English metaphor with strong national characteristics couldn't be translated to the same image with strong national features of our own. For instance, if we translate "in the country of blind, the one-eye man is king" into "shu zhong wu da jiang, Liao Hua chong xian feng", it would sound quite ridiculous to our Chinese readers. After all, it is really weird to find a traditional Chinese character "Liao Hua" in a totally foreign setting. In this case, we can just translate it into "shan zhong wu lao hu, hou zi cheng ba wang", using the image of "monkey" for instead. Image needs to be changed, but not be over-changed.

\subsection{Image-omitting translation}

The third one is omitting the image. It happens when the metaphor cannot be translated literally and at the same time, there is no equal metaphor in target language to replace the original one. In order to make the readers understand the translated version, translators have to omit the image and express clearly what the connotation is. Of course, this is the last situation the translators want to come across. After all, the image of metaphor is the color of the source language, and also the historical deposit in the culture. However, this kind of loss is necessary.

Taking the following sentence as example: "He stood a chinaman's chance of winning the election." In this sentence, if "chinaman's chance" is translated literally as "zhong guo ren de ji hui", it is not only confusing but also completely different from the original one. We might tend to associate the phrase "zhong guo ren de ji hui" with some big and international breakthrough for China. But actually, "chinaman's chance" originated from the Gold Rush in America. At that time, Chinese people have to wait for their turns of searching gold after those white people finishing their gold-finding job. Therefore, there is hardly any gold left for Chinese. The chance of getting rich is quite slim. Since the metaphor cannot be literally translated and furthermore, is strongly related to American history that means no equivalent Chinese metaphor to replace, the image then has to be omitted. So it is translated as "ta ji hu mei you ji hui ying de xuan ju".

Here is another example for translating from Chinese to English. "san ge ren pin zi shi zuo xia,sui bian tan le ji ju." (The three men sat down facing each other and began casually chatting). The expression of "pin zi shi"in the Chinese sentence is the description about the position of the seats by a vivid character. To make the English readers understand this expression, we have to give up the visualizing effect of "pin" as well as its metaphorical image. Other wise, we have to make out a note talking about a Chinese character, which looks quite strange to those English readers who have to make great effort to make it clear.

\section{CONCLUSION}

How to make a version of translation easy to understand and at the same time maintain the essence of source 
language has always been one of the difficult problems in translation research. Metaphor happens to be an expression fully loaded with the charm of a language. Looking back to the history of metaphor research, we can find that the understanding of metaphor is getting deeper and deeper. From a merely rhetoric device to a semantic phenomenon, and to a cognitive matter, the research of metaphor has already been an interdisciplinary field. Metaphor is no longer viewed as a merely linguistic issue, but a way of thought and a tool to cognize the world. As to the translation of metaphor, how to deal with that is still under research. Different translators would choose different ways when they come across the same sentence. In the process of translation, linguistic matter and cultural matter should be considered at the same time. Translators should try their best to fill the gap between two cultures and maintain the essence of the source language.

\section{ACKNOWLEDGMENT}

This work was supported by the Humanities and Social Sciences Fund from Sichuan Provincial Department of Education (14SB0065), the Education Reform Project of Chengdu University of Traditional Chinese Medicine (JGYB201955).

\section{REFERENCES}

[1] Encyclopedia Britannia On-line. (2017). Metaphor. http://www.britannica.com/eb/article-9052289/metapho $\mathrm{r}$

[2] Aristotle. (1970). Poetics. Gerald F. Else (trans.) The University of Michigan Press, Ann Arbor, MI. pp 57.

[3] Huang. J. M. (2007). The Translation of Metaphor. Journal of Qiongzhou University, 14(4): pp 107-108.

[4] [5] Lakoff, G \& Johnson, M. (2003). Metaphors We Live by. The University of Chicago Press, Chicago. pp 3-5.

[6] Burmakova, E.A. \& Marugina, N. I. (2014). Cognitive Approach to Metaphor Translation in Literary Discourse. Procedia - Social and Behavioral Sciences, 154: 527-533.

[7] Al-Hasnawi, Ali R. (2007). A Cognitive Approach to Translating Metaphors. Translation Watch Quarterly. 3(4): pp 62-74. 